\title{
A NOTE ON THE $R_{\infty}$ PROPERTY FOR GROUPS \\ $\operatorname{FAlt}(X) \leqslant G \leqslant \operatorname{Sym}(X)$
}

\author{
CHARLES GARNET COX
}

\begin{abstract}
Given a set $X$, the group $\operatorname{Sym}(X)$ consists of all bijective maps from $X$ to $X$, and $\operatorname{FSym}(X)$ is the subgroup of maps with finite support i.e. those that move only finitely many points in $X$. We describe the automorphism structure of groups $\operatorname{FSym}(X) \leqslant G \leqslant \operatorname{Sym}(X)$ and use this to state some conditions on $G$ for it to have the $R_{\infty}$ property. Our main results are that if $G$ is infinite, torsion, and $\operatorname{FSym}(X) \leqslant G \leqslant \operatorname{Sym}(X)$, then it has the $R_{\infty}$ property. Also, if $G$ is infinite and residually finite, then there is a set $X$ such that $G$ acts faithfully on $X$ and, using this action, $\langle G, \operatorname{FSym}(X)\rangle$ has the $R_{\infty}$ property. Finally we have a result for the Houghton groups, which are a family of groups we denote $H_{n}$, where $n \in \mathbb{N}$. We show that, given any $n \in \mathbb{N}$, any group commensurable to $H_{n}$ has the $R_{\infty}$ property.
\end{abstract}

\section{INTRODUCTION}

The notion of twisted conjugacy and its relationship to fixed point theory has attracted significant attention. For any group $G$ and any $\phi \in \operatorname{Aut}(G)$, we say that two elements $a, b \in G$ are $\phi$-twisted conjugate (denoted $a \sim_{\phi} b$ ) if there exists an $x \in G$ such that

$$
\left(x^{-1}\right) \phi a x=b .
$$

Notice that when $\phi=\mathrm{id}_{G}$ this becomes the equation for conjugacy. Now, given any $\phi \in \operatorname{Aut}(G)$, define the Reidemeister number of $\phi$, denoted $R(\phi)$, to be the number of $\phi$-twisted conjugacy classes in $G$. Thus $R\left(\mathrm{id}_{G}\right)$ records the number of conjugacy classes of $G$ and deciding whether this is infinite has been studied for some time (e.g. HNN49] where an infinite group with $R\left(\mathrm{id}_{G}\right)$ finite was constructed). We say that $G$ has the $R_{\infty}$ property if $R(\phi)=\infty$ for every $\phi \in \operatorname{Aut}(G)$.

Notation. For a non-empty set $X$, let $\operatorname{Sym}(X)$ denote the group of all permutations of $X$. Furthermore, let $\mathrm{FSym}(X)$ denote the group of all permutations of $X$ with finite support, and let $\operatorname{FAlt}(X)$ denote the group of all even permutations of $X$ with finite support.

A first example one may consider for the $R_{\infty}$ property is $\mathbb{Z}$. Although this has infinitely many conjugacy classes, the only non-trivial automorphism has Reidemeister number 2. Similarly, for any $m \in \mathbb{N}:=\{1,2, \ldots\}$, the automorphism $\psi$ of $\mathbb{Z}^{m}$ which sends $a$ to $a^{-1}$ for all $a \in \mathbb{Z}^{m}$ has Reidemeister number $2^{m}$. In [JLS17]

Date: June, 2018.

2010 Mathematics Subject Classification. Primary: 20E45, 20E36.

Key words and phrases. R infinity property, twisted conjugacy, twisted conjugacy classes, highly transitive groups, infinite torsion groups, Houghton's groups, commensurable groups. 
and GP16] however, the family of Houghton groups, which (for any $n \in \mathbb{N}$ ) are denoted $H_{n}$, act on $\{1, \ldots, n\} \times \mathbb{N}=: X_{n}$, and which lie in the short exact sequence

$$
1 \longrightarrow \operatorname{FSym}\left(X_{n}\right) \longrightarrow H_{n} \longrightarrow \mathbb{Z}^{n-1} \longrightarrow 1
$$

were shown to have the $R_{\infty}$ property. In this note we start with a simpler, more general proof of their theorem, and then develop this in various directions.

Definition 1.1. A group $G$ fully contains $\operatorname{FAlt}(X)$ if $\operatorname{FAlt}(X) \leqslant G \leqslant \operatorname{Sym}(X)$. Since we only wish to investigate infinite groups, we will always consider $X$ to be infinite. We do not, however, place any other cardinality assumptions on $X$.

Note that any Houghton group $H_{n}$ fully contains $\operatorname{FAlt}\left(X_{n}\right)$, but let us justify that this is a large class of groups, using a construction from [HO16. For any infinite group $G$, we have that $G \leqslant \operatorname{Sym}(X)$ for some $X$ (with the possibility that $X=G$ since $G$ can always be embedded into $\operatorname{Sym}(G)$ using a regular representation of $G)$. Then $\langle G, \operatorname{FAlt}(X)\rangle$ fully contains $\operatorname{FAlt}(X)$.

Conjecture 1.2. Let $G$ be an infinite group that acts faithfully on a set $X$. Then $\langle G, \operatorname{FAlt}(X)\rangle$ and $\langle G, \operatorname{FSym}(X)\rangle$ both have the $R_{\infty}$ property.

We make some progress with this conjecture. We first confirm it for the case where $G$ is torsion i.e. we show that, for any infinite set $X$ and any embedding of $\Psi: G \hookrightarrow \operatorname{Sym}(X),\langle(G) \Psi, \operatorname{FAlt}(X)\rangle$ has the $R_{\infty}$ property for any torsion group $G$. We then use this work to show that if $G$ is an infinite residually finite group, then there is a set $X$ on which $G$ acts faithfully and, using this action, $\langle G, \operatorname{FAlt}(X)\rangle$ has the $R_{\infty}$ property. We end by showing that, if $G$ is commensurable to a Houghton group $H_{n}$ (where $n \in \mathbb{N}$ ) then $G$ has the $R_{\infty}$ property.

Let us now describe these results more precisely, and better indicate the path that the paper takes. We start by describing the automorphism group for groups fully containing $\operatorname{FAlt}(X)$, so to approach twisted conjugacy.

Definition. A group $G$ is monolithic if it has a non-trivial normal subgroup that is contained in every non-trivial normal subgroup of $G$ i.e. if it has a minimal non-trivial normal subgroup.

Let $N_{\operatorname{Sym}(X)}(G):=\left\{\rho \in \operatorname{Sym}(X) \mid \rho^{-1} g \rho \in G\right.$ for every $\left.g \in G\right\}$, the normaliser of $G$ over $\operatorname{Sym}(X)$.

Proposition 1. (Lem. 2.4, Prop. 2.5, Rem. 2.6). Let $G$ fully contain FAlt $(X)$, where $X$ is infinite. Then $\operatorname{FAlt}(X)$ is characteristic in $G$, Aut $(G) \cong N_{\operatorname{Sym}(X)}(G)$, and $G$ is monolithic. Moreover, since elements of $\operatorname{Aut}(G)$ preserve the cycle type of elements of $G$, if $\operatorname{FSym}(X) \leqslant G$, then $\operatorname{FSym}(X)$ is characteristic in $G$.

The following well known lemma implies that if $G$ is a group with the $R_{\infty}$ property and $G$ acts faithfully on a set $X$, then $\langle G, \operatorname{FAlt}(X)\rangle$ and $\langle G, \operatorname{FSym}(X)\rangle$ have the $R_{\infty}$ property (since Proposition 1 states that FAlt $(X)$ and $\operatorname{FSym}(X)$ are characteristic in $\langle G, \operatorname{FAlt}(X)\rangle)$.

Lemma 1.3. MS14, Lem 2.1] For any short exact sequence of groups

$$
1 \longrightarrow D \longrightarrow E \longrightarrow F \longrightarrow 1
$$

if $D$ is characteristic in $E$ and $F$ has the $R_{\infty}$ property, then $E$ has the $R_{\infty}$ property.

We then work with arguments using cycle type (using that the conjugacy classes of $\operatorname{Sym}(X)$ are well know: each consists of all elements of the same cycle type). 
Definition. Let $g \in \operatorname{Sym}(X)$. Then an orbit of $g$ is $\left\{x g^{d} \mid d \in \mathbb{Z}\right\}$ where $x \in X$. Also, $g$ has an infinite orbit if there is a $y \in X$ such that $\left\{y g^{d} \mid d \in \mathbb{Z}\right\}$ is infinite.

Proposition 3.4. Let $G$ fully contain $\operatorname{FAlt}(X)$, where $X$ is an infinite set. If for every $\rho \in N_{\operatorname{Sym}(X)}(G)$ there is an $s \in \mathbb{N}$ such that $\rho$ has finitely many orbits of size $s$, then $G$ has the $R_{\infty}$ property.

From the structure of $\operatorname{Aut}\left(H_{n}\right)$, where $H_{n}$ denotes the $n^{\text {th }}$ Houghton group, Proposition 3.4 immediately yields that, for any $n \geqslant 2, H_{n}$ has the $R_{\infty}$ property.

Corollary 3.6. Let $G$ fully contain $\operatorname{FAlt}(X)$, where $X$ is an infinite set. If for every $g \in G, g$ does not have an infinite orbit, then $G$ has the $R_{\infty}$ property.

Clearly torsion groups satisfy Corollary 3.6 .

Corollary 3.7. Let $G$ be an infinite torsion group which fully contains $\operatorname{FAlt}(X)$. Then $G$ has the $R_{\infty}$ property.

This means that any torsion group $T$ can be embedded into an infinite torsion group (of any cardinality greater than or equal to $|T|$ ) which has the $R_{\infty}$ property. It is in fact easy to construct an uncountable family of such groups.

Corollary 3.11. There exist uncountably many countable torsion groups which have the $R_{\infty}$ property.

This result can be strengthened by using an already known family of countable, finitely generated, torsion groups.

Corollary 3.12, There exist uncountably many finitely generated torsion groups which have the $R_{\infty}$ property.

Residually finite groups are exactly those that have a faithful action on their finite quotients. This action therefore only has finite orbits, meaning that Corollary 3.6 applies.

Corollary 3.15. Let $G$ be an infinite residually finite group, and $X$ be the union of the finite quotients of $G$. Then, using this action, $\langle G, \operatorname{FAlt}(X)\rangle$ has the $R_{\infty}$ property.

A few conventions will be used throughout this note:

i) we shall always work with right actions;

ii) unless specified, $X$ will refer to an infinite set;

iii) we shall always consider elements from $\operatorname{Sym}(X)$ to be written in disjoint cycle notation;

iv) for all of the results in this note, the same proofs can be used if FAlt is replaced with FSym.

Remark. Let $g \in \operatorname{Sym}(X)$. We shall say 'a cycle of $g$ ' to refer, for some $x \in X$, to an orbit $\left\{x g^{d} \mid d \in \mathbb{Z}\right\}$. If there is an $x \in X$ such that this set is infinite, then this is an infinite cycle of $g$ and $g$ contains an infinite cycle. If there is an $x \in X$ such that this set has cardinality $r$, then this is an $r$-cycle of $g$ and $g$ contains an $r$-cycle. If, for some $s \in \mathbb{N}$, there are only finitely many $x \in X$ such that $\left|\left\{x g^{d} \mid d \in \mathbb{Z}\right\}\right|=s$, then we shall say that $g$ has finitely many s-cycles. Similarly $g$ may have finitely many infinite cycles. 
Acknowledgements. I thank the authors of [JLS17] and GP16, whose papers drew my attention to the $R_{\infty}$ property. I thank my supervisor Armando Martino for his guidance and encouragement. I thank Hector Durham, of the University of Southampton, for the numerous interesting discussions, especially those regarding monolithic groups. I thank Motiejus Valiunas, also of the University of Southampton, for his suggestion to choose $X$ as the union of finite quotients for an action with only finite orbits for residually finite groups, the main ingredient of Corollary 3.15. Finally I thank the referee for their useful comments.

\section{Preliminary observations}

The groups FAlt $(X), \operatorname{FSym}(X)$, and $\operatorname{Sym}(X)$ often arise when considering permutation groups (see, for example, Cam99] and [DM96]). Note that any countable group can be considered as a subgroup of $\operatorname{Sym}(X)$ where $X$ is countable (for example set $X:=G$ and use the regular representation of $G$ ). Let us start by recalling some elementary observations about FAlt.

Lemma 2.1. If $G$ fully contains $\operatorname{FAlt}(X)$ where $X$ is infinite, then $G$ is centreless.

Lemma 2.2. For any infinite set $X, \operatorname{FAlt}(X)$ is generated by $S$, where $S$ is the set of all 3-cycles with support in $X$.

Proof. Using $S$ we can produce any element which is a product of two 2-cycles (for example choose $\left(a_{1} a_{2}\right)\left(a_{2} b_{1}\right)$ and $\left(a_{2} b_{1}\right)\left(b_{1} b_{2}\right)$ whose product is $\left.\left(a_{1} a_{2}\right)\left(b_{1} b_{2}\right)\right)$. Now, given an element $\sigma \in \operatorname{FAlt}(X)$, write $\sigma$ as a product of 2-cycles. By definition this product will consist of an even number of 2-cycles. Now, each pair of 2-cycles will either be: trivial; a 3-cycle; or a product of two 2-cycles.

For any infinite set $X$ we therefore have that $|X|=|S|=|\operatorname{FAlt}(X)|$. Moreover FAlt $(X)$ is an index 2 subgroup of $\operatorname{FSym}(X)$, and so for any infinite set $X$ we also have that $|X|=|\operatorname{FSym}(X)|$.

Lemma 2.3. For any infinite set $X, \operatorname{FAlt}(X)$ is simple.

Proof. Assume that $1 \neq \sigma \in N$, a non-trivial normal subgroup of $\operatorname{FAlt}(X)$. Then $\sigma \in A_{n}$ where $n \geqslant 5$. But $A_{n} \cap N \unlhd A_{n}$, and (since $N$ is non-trivial and $A_{n}$ is simple for $n \geqslant 5$ ) we have that $N \cap A_{n}=A_{n}$. Thus $N$ contains a 3 -cycle and so $N=\operatorname{FAlt}(X)$ by the previous lemma.

Note that no infinite simple group can be residually finite, and so if $G$ fully contains $\operatorname{FAlt}(X)$, then $G$ is not residually finite. Also, given any infinite set $X$, any group $G$ fully containing $\operatorname{FAlt}(X)$ will have $\operatorname{FAlt}(X)$ as a normal subgroup. Thus, unless $G=\operatorname{FAlt}(X), G$ will not be simple.

Notation. Let $G \leqslant \operatorname{Sym}(X)$. For any given $\rho \in N_{\operatorname{Sym}(X)}(G)$, let $\phi_{\rho}$ denote the automorphism of $G$ induced by conjugation by $\rho$ i.e. $(g) \phi_{\rho}:=\rho^{-1} g \rho$ for all $g \in G$.

The three groups $\operatorname{FAlt}(X), \operatorname{FSym}(X), \operatorname{Sym}(X)$ have the property that

$$
N_{\operatorname{Sym}(X)}(G) \rightarrow \operatorname{Aut}(G), \rho \mapsto \phi_{\rho} \text { is an isomorphism. }
$$

This means that $\operatorname{Aut}(\operatorname{FAlt}(X)) \cong N_{\operatorname{Sym}(X)}(\operatorname{FAlt}(X))=\operatorname{Sym}(X) \cong \operatorname{Aut}(\operatorname{FSym}(X))$ and that $\operatorname{FAlt}(X)$ is characteristic in $\operatorname{FSym}(X)$ which is characteristic in $\operatorname{Sym}(X)$. Our first aim is to show that any group $G$ fully containing $\operatorname{FAlt}(X)$ satisfies (2). We do this by showing that $\operatorname{FAlt}(X)$ is characteristic in such a $G$ and then apply the following lemma. 
Lemma 2.4. Let $G \leqslant \operatorname{Sym}(X)$ and $\operatorname{FAlt}(X)$ be a characteristic subgroup of $G$. Then $N_{\operatorname{Sym}(X)}(G) \cong_{\Psi} \operatorname{Aut}(G)$ where $\Psi: \rho \mapsto \phi_{\rho}$.

Proof. Running the proof of GP16, Cor. 3.3] using 3-cycles rather than 2-cycles yields the result.

For any group $G$ satisfying (2), we may use the following reformulation of twisted conjugacy, which has been used extensively by many authors working with the $R_{\infty}$ property. Recall that $\phi_{\rho}$ denotes the automorphism induced by conjugation by $\rho \in \operatorname{Sym}(X)$. Thus,

$$
\left(x^{-1}\right) \phi_{\rho} a x=b \Rightarrow \rho^{-1}\left(x^{-1}\right) \rho a x=b \Rightarrow x^{-1} \rho a x=\rho b .
$$

We may then show that $R\left(\phi_{\rho}\right)=\infty$ by finding a set of elements $\left\{a_{k} \in G \mid k \in \mathbb{N}\right\}$ such that

$$
\rho a_{i} \sim_{G} \rho a_{j} \Leftrightarrow i=j .
$$

This is because, if such a set of elements exist, then each $a_{k}$ lies in a distinct $\phi_{\rho}$-twisted conjugacy class, and so $R\left(\phi_{\rho}\right)=\infty$. Thus showing, for each $\rho \in N_{\operatorname{Sym}(X)}(G)$, that there exists a set of elements $\left\{a_{k} \in G \mid k \in \mathbb{N}\right\}$ where (4) holds is sufficient to show that $G$ has the $R_{\infty}$ property.

Proposition 2.5. If $G$ fully contains $\operatorname{FAlt}(X)$, then $\operatorname{FAlt}(X)$ is a unique minimal normal subgroup of $G$. Moreover $\operatorname{FAlt}(X)$ is a characteristic subgroup of $G$.

Proof. We first show that $\operatorname{FAlt}(X)$ is a unique minimal normal subgroup of $G$, known as the monolithic property. Clearly $\operatorname{FAlt}(X)$ is normal in $G$, since it is normal in $\operatorname{Sym}(X)$ (conjugation in $\operatorname{Sym}(X)$ preserves cycle type).

Consider $N \unlhd G$. We have $N \cap \operatorname{FAlt}(X) \unlhd \operatorname{FAlt}(X)$, and since $\operatorname{FAlt}(X)$ is simple, $N \cap \operatorname{FAlt}(X)$ must either be trivial or $\operatorname{FAlt}(X)$. Let $g \in N \backslash\{1\}$. This must either: be in $\operatorname{FSym}(X)$; contain infinitely many finite cycles; or contain an infinite cycle. We now show that there exists a $\sigma \in \operatorname{FAlt}(X)$ such that $\sigma^{-1} g \sigma g^{-1} \in \operatorname{FAlt}(X) \backslash\{1\}$. Since $N$ is normal in $G, g$ and $\sigma^{-1} g \sigma$ are in $N$ and so this will prove the claim. For the case where $g \in \mathrm{FSym}(X)$, choose $\sigma$ so that $\sigma^{-1} g \sigma$ and $g$ have disjoint supports. For the case where $g$ contains infinitely many finite cycles, pick 4 distinct cycles (each of length greater than 1) of $g$ and points $b_{1}, b_{2}, b_{3}, b_{4}$ : one from each cycle. A suitable $\sigma$ is then $\left(b_{1} b_{2}\right)\left(b_{3} b_{4}\right)$. Finally, assume that $g$ contains an infinite cycle. Let $x_{0} \in X$ lie in some infinite cycle of $g$, and for every $i \in \mathbb{Z}$ let $x_{i}:=x_{0} g^{i}$. Let $a:=\left(\begin{array}{lllllll}\ldots x_{-3} & x_{-2} & x_{-1} & x_{0} & x_{1} & x_{2} & x_{3}\end{array} \ldots\right)$ and let $\mu:=\left(\begin{array}{lll}x_{-1} & x_{0} & x_{1}\end{array}\right)$. Straightforward computation shows that $\mu^{-1} a \mu a^{-1}=\left(x_{-2} x_{-1} x_{1}\right)$. Moreover, since $\mu$ commutes with $g a^{-1}$, we have that $\mu^{-1} g \mu g^{-1}=\left(x_{-2} x_{-1} x_{1}\right)$. Thus $\mu$ is a suitable candidate for $\sigma$ in this case.

Now, let $\phi \in \operatorname{Aut}(G)$ and consider $\operatorname{FAlt}(X) \cap(\operatorname{FAlt}(X)) \phi$. As above, this must be trivial or FAlt $(X)$. If it were trivial, this would contradict the uniqueness of FAlt $(X)$ as a minimal, non-trivial, normal subgroup in $G$, and hence $\operatorname{FAlt}(X)$ is characteristic in $G$.

Remark 2.6. We may use Lemma 2.4 and Proposition 2.5 to prove that all automorphisms of $\operatorname{Sym}(X)$ are inner. Also, consider if $\operatorname{FSym}(X) \leqslant G \leqslant \operatorname{Sym}(X)$. Then, for all $\rho \in N_{\operatorname{Sym}(X)}(G)$ and all $g \in \operatorname{FSym}(X)$, we have that $(g) \phi_{\rho}$ has the same cycle type as $g$. Thus $\mathrm{FSym}(X)$ is characteristic in $G$. 
We are now ready to produce conditions on the cycle type of elements in $G$ and in $N_{\text {Sym }(X)}(G)$ for automorphisms to have infinite Reidemeister number. In order to do this we will use the condition equivalent to showing that $R\left(\phi_{\rho}\right)=\infty$ (labelled (4) above) and well known facts about $\operatorname{Sym}(X)$ regarding cycle type.

\section{Results using facts about conjugacy in Sym}

Lemma 3.1. Let $Y$ be an infinite set and $X$ be an infinite subset of $Y$. If $\operatorname{FAlt}(X)$ is a subgroup of $G \leqslant \operatorname{Sym}(Y)$, then $R\left(\operatorname{id}_{G}\right)=\infty$.

Proof. We produce an infinite family of elements in $G$ which all lie in distinct conjugacy classes. We have the equation $g^{-1} a g=b$. Conjugation by elements of $G$ cannot change the cycle type of elements of $\operatorname{Sym}(X) \leqslant \operatorname{Sym}(Y)$. Thus choosing $a_{k}$ to be a cycle of length $2 k+1$ (or any infinite family of elements of $\operatorname{FAlt}(X)$ with distinct cycle types) proves the claim.

The following is well known.

Lemma 3.2. Let $G$ be any group. Then, for any $\psi \in \operatorname{Aut}(G)$ and $\phi \in \operatorname{Inn}(G)$, we have that $R(\psi \phi)=R(\psi)$.

Lemma 3.3. Let $G$ be a group with subgroup $\operatorname{FAlt}(X)$, where $X$ is an infinite set, and with $\operatorname{Aut}(G)=\operatorname{Inn}(G)$. Then $G$ has the $R_{\infty}$ property.

Proof. Let $\phi \in \operatorname{Aut}(G)$. By assumption $\operatorname{Aut}(G)=\operatorname{Inn}(G)$. Therefore, by the previous lemma, $R(\phi)=R\left(\operatorname{id}_{G}\right)$. Now $R\left(\operatorname{id}_{G}\right)=\infty$ by Lemma 3.1.

Lemma 3.3 implies that, for any infinite set $X, \operatorname{Sym}(X)$ has the $R_{\infty}$ property.

Notation. For any $g \in \operatorname{Sym}(X)$ and $x \in X$, let $\mathcal{O}_{x}(g):=\left\{x g^{d}: d \in \mathbb{Z}\right\}$. Also, let $\eta_{r}(g):=\left|\left\{x \in X:\left|\mathcal{O}_{x}(g)\right|=r\right\}\right| / r$, the number of $r$-cycles in $g$. We shall use $\eta_{1}(g)$ to denote the number of fixed points of $g$ and $\eta_{\infty}(g)$ to denote the number of distinct infinite orbits induced by $g$. If any of these values is infinite then, since our arguments will be unaffected by the size of this infinity, we shall write $\eta_{r}(g)=\infty$.

From the previous section, for any group fully containing $\operatorname{FAlt}(X)$ we have that the map $\Psi: N_{\operatorname{Sym}(X)}(G) \rightarrow \operatorname{Aut}(G), \rho \mapsto \phi_{\rho}$ is an isomorphism. We may therefore consider elements of $\operatorname{Aut}(G)$ as elements of $\operatorname{Sym}(X)$.

Proposition 3.4. Let $G$ fully contain FAlt $(X)$, where $X$ is an infinite set, and let $\rho \in N_{\operatorname{Sym}(X)}(G)$. If there is an $r \in \mathbb{N}$ such that $\eta_{r}(\rho)<\infty$, then $R\left(\phi_{\rho}\right)=\infty$.

Proof. We shall work with the reformulation of twisted conjugacy in (4) above and argue for any $\rho \in N_{\operatorname{Sym}(X)}(G)$ using three cases. Let $s \in \mathbb{N}$ be the smallest number such that $\eta_{s}(\rho)$ is finite.

Case A: $s=1$ and $\eta_{\infty}(\rho)>0$. As with the proof of Proposition 2.5, let $x_{0}$ lie in an infinite cycle of $\rho$ and, for each $i \in \mathbb{Z}$, let $x_{i}:=x_{0} g^{i}$. For each $k \in \mathbb{N}$, let

$$
a_{k}:=\prod_{i=0}^{k-1}\left(\begin{array}{ll}
x_{2 i} & x_{2 i+1}
\end{array}\right) .
$$

The set of elements lying in disjoint $\phi_{\rho}$-twisted conjugacy classes is then given by $\left\{a_{2 k} \mid k \in \mathbb{N}\right\} \subset \operatorname{FAlt}(X)$. This is because $\eta_{1}\left(\rho a_{k}\right)$ is finite for all $k \in \mathbb{N}$, and is strictly increasing as a function of $k$. Thus, if $i \neq j$, the elements $\rho a_{i}$ and $\rho a_{j}$ have a different number of fixed points and hence are not conjugate in $G \leqslant \operatorname{Sym}(X)$. 
Case B: $s=1$ and $\eta_{\infty}(\rho)=0$. Since $\rho$ has finitely many fixed points and no infinite cycles, $\rho$ contains infinitely many finite cycles. Thus $\rho$ has infinitely many odd length cycles or infinitely many even length cycles. First assume that $\rho$ has infinitely many odd length cycles and index a countably infinite subset of these by the natural numbers. Let $\rho=\rho^{\prime} \prod_{i \in \mathbb{N}} \rho_{i}$, where each $\rho_{i}$ is a finite cycle of odd length and $\rho^{\prime} \in \operatorname{Sym}(X)$ has cycles with disjoint support from all of the $\rho_{i}$ 's. Now, for any $m \in \mathbb{N}, \rho\left(\rho_{m}\right)^{-1}$ has more fixed points than $\rho$. Defining

$$
a_{k}:=\prod_{i=1}^{k} \rho_{i}^{-1} \in \operatorname{FAlt}(X)
$$

means that $i<j \Rightarrow \eta_{1}\left(\rho a_{i}\right)<\eta_{1}\left(\rho a_{j}\right)$ and so $\left\{a_{k} \mid k \in \mathbb{N}\right\}$ provides our infinite family of elements which are pairwise not $\phi_{\rho}$-twisted conjugate. Similarly, if $\rho$ has infinitely many even length cycles, complete the same construction with $\rho=$ $\rho^{\prime} \prod_{i \in \mathbb{N}} \rho_{i}$ where each $\rho_{i}$ is a finite cycle of $\rho$ of even length and $\rho^{\prime} \in \operatorname{Sym}(X)$ has cycles with disjoint support from all of the $\rho_{i}$ 's. Then $\left\{a_{2 k} \mid k \in \mathbb{N}\right\}$ provides the infinite family in $\operatorname{FAlt}(X)$.

Case C: $s>1$. All we shall use is that $\rho$ has infinitely many fixed points. For any $k \in \mathbb{N}$, let $a_{k}$ consist of $2 k s$-cycles such that $\operatorname{supp}\left(a_{k}\right) \subset X \backslash \operatorname{supp}(\rho)$. We then have, for all $k \in \mathbb{N}$ : that $a_{k} \in \operatorname{FAlt}(X)$; that $\eta_{s}\left(\rho a_{k}\right)$ is finite; and that $\eta_{s}\left(\rho a_{k}\right)$ is strictly increasing as a function of $k$.

Proposition 3.5. Let $a, b \in \operatorname{Sym}(X), \operatorname{supp}(b) \subsetneq \operatorname{supp}(a)$, and $g \in \operatorname{Sym}(X)$ satisfy $g^{-1} a g=b$. Then $\eta_{\infty}(g)>0$.

Proof. We assume, for a contradiction, that $\eta_{\infty}(g)=0$. Since $g^{-1} a g=b, g$ must restrict to a bijection from $\operatorname{supp}(a)$ to $\operatorname{supp}(b)$ i.e.

$$
(\operatorname{supp}(a) \cup \operatorname{supp}(b)) \backslash(\operatorname{supp}(a) \cap \operatorname{supp}(b)) \subseteq \operatorname{supp}(g)
$$

which from our hypotheses implies that

$$
\operatorname{supp}(a) \backslash \operatorname{supp}(b) \subseteq \operatorname{supp}(g)
$$

where $\operatorname{supp}(a) \backslash \operatorname{supp}(b) \neq \emptyset$ since $\operatorname{supp}(b) \neq \operatorname{supp}(a)$. Thus $g$ sends some $n \in$ $\operatorname{supp}(a) \backslash \operatorname{supp}(b)$ to some $m \in \operatorname{supp}(b)$. Now, since all of the cycles in $g$ are finite, there is a $k \in \mathbb{N}$ such that $(n) g^{k}=n$. Therefore $g$ sends a point in $\operatorname{supp}(b)$ to a point in $X \backslash \operatorname{supp}(b)$. This would mean that $\operatorname{supp}\left(g^{-1} a g\right) \cap(X \backslash \operatorname{supp}(b)) \neq \emptyset$ and that $g^{-1} a g$ and $b$ have different supports, a contradiction.

Corollary 3.6. Let $G$ be a group fully containing $\operatorname{FAlt}(X)$. If $\eta_{\infty}(g)=0$ for all $g \in G$, then $G$ has the $R_{\infty}$ property.

Proof. By Proposition 3.4 if $\phi_{\rho} \in \operatorname{Aut}(G)$ has $\eta_{s}(\rho)<\infty$ for some $s \in \mathbb{N}$, then $R\left(\phi_{\rho}\right)=\infty$. We may therefore assume that $\eta_{r}(\rho)=\infty$ for all $r \in \mathbb{N}$. This implies that $X \backslash \operatorname{supp}(\rho)$ is an infinite set.

Our aim is to show that there is an infinite set of elements in $G$ which are not $\phi_{\rho}$-twisted conjugate. Let $b_{0}:=1$, the identity element of $G$. For each $k \in \mathbb{N}$, let $b_{k}:=b_{k}^{\prime} b_{k-1}$ where $\eta_{2}\left(b_{k}^{\prime}\right)=2,\left|\operatorname{supp}\left(b_{k}^{\prime}\right)\right|=4, \operatorname{supp}\left(b_{k}^{\prime}\right) \subset X \backslash \operatorname{supp}(\rho)$, and $\operatorname{supp}\left(b_{k}^{\prime}\right) \cap \operatorname{supp}\left(b_{k-1}\right)=\emptyset$. Thus, for each $k \in \mathbb{N}, b_{k} \in \operatorname{FAlt}(X)$ and $\eta_{2}\left(b_{k}\right)=2 k$. If $i<j$, then $\operatorname{supp}\left(b_{i}\right) \subsetneq \operatorname{supp}\left(b_{j}\right)$ and so $\operatorname{supp}\left(\rho b_{i}\right) \subsetneq \operatorname{supp}\left(\rho b_{j}\right)$. Since $\eta_{\infty}(g)=0$ for all $g \in G$, Proposition 3.5 implies that not two elements in $\left\{\rho b_{k} \mid k \in \mathbb{N}\right\}$ are conjugate in $G$ i.e. $R\left(\phi_{\rho}\right)=\infty$. 
Notice that this provides an alternative proof to [JLS17] and [GP16] that FSym $(X)$ has the $R_{\infty}$ property. We also have the following.

Corollary 3.7. Let $G$ be an infinite torsion group which fully contains $\operatorname{FAlt}(X)$. Then $G$ has the $R_{\infty}$ property.

Lemma 3.8. Let $G \leqslant \operatorname{Sym}(X)$ be torsion. Then $\langle G, \operatorname{FAlt}(X)\rangle$ is also torsion.

Proof. Consider an element $\sigma g$ where $\sigma \in \operatorname{FSym}(X)$ and $g \in G$. It suffices to show that $\sigma g$ is torsion. Fix a $k \in \mathbb{N}$ such that $\operatorname{supp}(\sigma)$ has trivial intersection with all orbits of $g$ of size $r>k$. Since $G$ is torsion, the order of $g$ is one such $k$ (but since $|\operatorname{supp}(\sigma)|<\infty$, we could find a $k$ using only the assumption that all orbits of all elements of $G$ are finite). Let

$$
F:=\bigcup_{1 \leqslant i \leqslant k} \operatorname{supp}\left(g^{-i} \sigma g^{i}\right)
$$

Now $|F| \leqslant k|\operatorname{supp}(\sigma)|<\infty, \sigma g$ restricts to a bijection on $X \backslash F$, the elements $\sigma g$ and $g$ induce the same permutation on $X \backslash F$, and the orbits of $g$ within $X \backslash F$ are of bounded size (since otherwise $g$ cannot be torsion). Hence the orbits of $\sigma g$ within $X$ are of bounded size and so $\sigma g$ is torsion.

Remark 3.9. A consequence of this proof is that if all elements of $G \leqslant \operatorname{Sym}(X)$ have only finite orbits, then the elements of $\langle G, \operatorname{FAlt}(X)\rangle$ also only have finite orbits.

Corollary 3.10. Let $G$ be a torsion group. For every infinite $\alpha \geqslant|G|$, there exists a torsion group $H_{\alpha}$ of cardinality $\alpha$ which has the $R_{\infty}$ property and contains an isomorphic copy of $G$.

Proof. Let $G$ be torsion, $\hat{G}$ denote the right regular representation of $G$, and let $\alpha \geqslant G$ if $G$ is infinite and $\alpha \geqslant|\mathbb{Z}|$ otherwise. Then there is a set $Y_{\alpha}$ such that $\left|Y_{\alpha}\right|=\alpha$. Also $\hat{G} \leqslant \operatorname{Sym}(G) \hookrightarrow \operatorname{Sym}\left(G \sqcup Y_{\alpha}\right)$ via the natural inclusion of the set $G$ into the set $G \sqcup Y_{\alpha}$. Let $G_{\alpha}$ denote the image of $\hat{G}$ in $\operatorname{Sym}\left(G \sqcup Y_{\alpha}\right)$, using the restriction of this map. Now $H_{\alpha}:=\left\langle G_{\alpha}, \operatorname{FAlt}\left(G \sqcup Y_{\alpha}\right)\right\rangle$ has cardinality $\alpha$. Moreover it is torsion by Lemma 3.8 and so has the $R_{\infty}$ property by Corollary 3.7

There are also groups which are not torsion and have no infinite cycles. Consider an element $\rho \in \operatorname{Sym}(X)$ with $\eta_{r}(\rho)$ non-zero for infinitely many $r \in \mathbb{N}$. Then $\rho$ has infinite order, but need not contain an infinite cycle. Therefore $\rho$ generates an infinite cyclic group, but $\langle\rho, \operatorname{FSym}(X)\rangle$ is not finitely generated. This is an interesting example since $\mathrm{FSym}(\mathbb{Z}) \rtimes \mathbb{Z}$, which also consists of the group FSym together with a single element of infinite order, is 2-generated (being the second Houghton group $H_{2}$ ). For another example, consider $G=\prod_{i \in \mathbb{N}} C_{2}$. This can be seen as a subgroup $G_{1}$ of $\operatorname{Sym}(\{1,2\} \times \mathbb{N})$ where the $i$ th $C_{2}$ transposes the points $(1, i)$ and $(2, i)$ and fixes all other points of $\{1,2\} \times \mathbb{N}$. Now $\prod_{i \in \mathbb{N}} C_{2} \cong \bigoplus_{i \in \mathbb{R}} C_{2}$ (both are vector spaces of rank $|\mathbb{R}|$ over $F_{2}$ ) and so $G$ can also be seen as a subgroup $G_{2}$ of $\operatorname{Sym}(\{1,2\} \times \mathbb{R})$ with generators $g_{i}$ (for each $i \in \mathbb{R}$ ) that transpose the points $(1, i)$ and $(2, i)$ and fix all other points of $\{1,2\} \times \mathbb{R}$. But $\left\langle G_{1}, \operatorname{FAlt}(\{1,2\} \times\right.$ $\mathbb{N})\rangle \neq\left\langle G_{2}, \operatorname{FAlt}(\{1,2\} \times \mathbb{R})\right\rangle$, since $\left\langle G_{1}, \operatorname{FAlt}(\{1,2\} \times \mathbb{N})\right\rangle \leqslant \operatorname{Sym}(\{1,2\} \times \mathbb{N})$ and $\operatorname{FAlt}(\{1,2\} \times \mathbb{R})$ does not embed into $\operatorname{Sym}(\{1,2\} \times \mathbb{N})$ by $[$ BH16] .

Corollary 3.11. There exist uncountably many countable torsion groups which have the $R_{\infty}$ property. 
Proof. We will work within $\operatorname{Sym}(\mathbb{N} \times \mathbb{N})$. For each $n \geqslant 2$, define

$$
\phi^{(n)}: C_{n} \hookrightarrow \operatorname{Sym}(\mathbb{N} \times \mathbb{N}),(1 \ldots n) \mapsto \rho_{n}
$$

where $\operatorname{supp}\left(\rho_{n}\right)=\{(m, n) \mid m \in \mathbb{N}\}$ and

$$
(m, n) \rho_{n}:= \begin{cases}(m-n+1, n) & \text { if } m \equiv 0 \bmod n \\ (m+1, n) & \text { otherwise }\end{cases}
$$

i.e. $\rho_{n}$ consists of $n$-cycles 'all the way along' the $n^{\text {th }}$ copy of $\mathbb{N}$.

Let $\mathbb{P}$ denote the set of all prime numbers. Then, for any subset $S \subseteq \mathbb{P}$, let $G_{S}:=\bigoplus_{p \in S} C_{p}$. Note that there are uncountably many choices for $S$. Also,

$$
\bigoplus_{p \in S} C_{p} \hookrightarrow \operatorname{Sym}(\mathbb{N} \times \mathbb{N})
$$

by using the maps $\phi^{(n)}$ defined above. For any $S \subseteq \mathbb{P}$, let $\tilde{G}_{S}:=\left\langle G_{S}, \operatorname{FAlt}(\mathbb{N} \times \mathbb{N})\right\rangle$, which fully contains $\operatorname{FAlt}(\mathbb{N} \times \mathbb{N})$ and, by Lemma 3.8 , is torsion. Hence Corollary 3.7 applies to $\tilde{G}_{S}$ and it has the $R_{\infty}$ property. Our final aim is therefore to show that if $S \neq S^{\prime}$, then $\tilde{G}_{S}$ and $\tilde{G}_{S^{\prime}}$ are not isomorphic. By Proposition 2.5, $\tilde{G}_{S}$ and $\tilde{G}_{S^{\prime}}$ each have FAlt $(\mathbb{N} \times \mathbb{N})$ as a unique minimal normal subgroup. Since $G_{S}$ and $G_{S^{\prime}}$ contain no non-trivial elements of finite support,

$$
\tilde{G}_{S} / \operatorname{FAlt}(\mathbb{N} \times \mathbb{N}) \cong G_{S} \text { and } \tilde{G}_{S^{\prime}} / \operatorname{FAlt}(\mathbb{N} \times \mathbb{N}) \cong G_{S^{\prime}} .
$$

Hence if $\tilde{G}_{S}$ and $\tilde{G}_{S^{\prime}}$ are isomorphic, then $G_{S}$ and $G_{S^{\prime}}$ are isomorphic. But since $S \neq S^{\prime}$, there is a $p \in \mathbb{P}$ in one set that is not in the other. Without loss of generality let $p \in S \backslash S^{\prime}$. By construction, $G_{S}$ has $p$-torsion but $G_{S^{\prime}}$ does not. Hence $\tilde{G}_{S} \neq \tilde{G}_{S^{\prime}}$.

Corollary 3.12. There exist uncountably many finitely generated torsion groups which have the $R_{\infty}$ property.

Proof. In Ols82 the Tarski monsters, an uncountable family of finitely generated infinite p-groups, are described. Let $M_{1}$ and $M_{2}$ be non-isomorphic Tarski monsters. For any group $G$, let $\hat{G}$ denote the right regular representation of $G$ and let $\tilde{G}:=$ $\langle\hat{G}, \operatorname{FSym}(G)\rangle$. By Lemma 3.8, $\tilde{M}_{1}$ and $\tilde{M}_{2}$ are torsion. By [HO16, Prop 5.10], $\tilde{M}_{1}$ and $\tilde{M}_{2}$ are finitely generated. Moreover $\tilde{M}_{1} \neq \tilde{M}_{2}$ since they are both monolithic (by Proposition 2.5) but if we quotient by this unique minimal normal subgroup then we obtain non-isomorphic groups.

There are many equivalent definitions of the following.

Definition 3.13. A group $G$ is residually finite if for each non-trivial element $g \in G$ there exists a finite group $F_{g}$ and a homomorphism $\phi_{g}: G \rightarrow F_{g}$ such that $(g) \phi_{g} \neq 1$.

It is the following well known reformulation that shall be of use to us.

Lemma 3.14. A group $G$ is residually finite if and only if it can be embedded inside the direct product of a family of finite groups. Moreover the family comprises of the finite quotients of $G$.

Corollary 3.15. Let $G$ be an infinite residually finite group, and $X$ be the union of the finite quotients of $G$. Then, using this action, $\langle G, \operatorname{FAlt}(X)\rangle$ has the $R_{\infty}$ property. 
Proof. Since $G$ is residually finite, it can be embedded inside the direct product of a family of finite groups (which are those groups appearing as finite quotients of $G)$. Therefore any element $g \in G$ has only finite orbits, and by Remark 3.9, any element in $\langle G, \operatorname{FAlt}(X)\rangle$ also only has finite orbits. Hence Corollary 3.6 applies, and $\langle G, \operatorname{FAlt}(X)\rangle$ has the $R_{\infty}$ property.

\section{The $R_{\infty}$ Property And COMmensurable Groups}

This final section involves results for commensurable groups.

Notation. Let $N \unlhd_{f} G$ denote that $N$ is normal and finite index in $G$.

Definition 4.1. Let $G$ and $H$ be groups. We say that $G$ is commensurable to $H$ if and only if there exist $N_{G} \cong N_{H}$ with $N_{G} \unlhd_{f} G$ and $N_{H} \unlhd_{f} H$. Note that if $G$ is finitely generated then so is $N_{G}$, which then implies that $H$ is finitely generated.

We will work towards Theorem 4.6 which applies to the Houghton groups, a family of groups $H_{n}$ indexed over $\mathbb{N}$ where, for each $n \in \mathbb{N}, H_{n}$ acts on a set $X_{n}$ and $\operatorname{FSym}\left(X_{n}\right) \leqslant H_{n} \leqslant \operatorname{Sym}\left(X_{n}\right)$. Each group $H_{n}$ therefore fully contains FAlt $\left(X_{n}\right)$. These were first introduced in Hou78, but we rely heavily on Cox17] where an introduction to these groups can be found and a description, for all $n \geqslant 2$, of the structure of the automorphism group for all finite index subgroups of $H_{n}$ is given. We start with three well known results.

Lemma 4.2. If $H \leqslant_{f} G$, then $\exists N \leqslant_{f} H$ which is normal in $G$.

Proof. Let $H$ have index $n$ in $G$ and let $N:=\bigcap_{g \in G}\left(g^{-1} H g\right)$. Then $G$ acts on $H \backslash G$ by right multiplication, and so there is a homomorphism $\phi: G \rightarrow S_{n}$. Now $h \in \operatorname{ker}(\phi)$ if,

$$
\begin{aligned}
& H g h=H g \text { for all } g \in G \\
\Leftrightarrow & g h g^{-1} \in H \text { for all } g \in G \\
\Leftrightarrow & h \in g^{-1} H g \text { for all } g \in G .
\end{aligned}
$$

Hence $\operatorname{ker}(\phi)=N$ and $N$ is normal. Moreover $G / \operatorname{ker}(\phi) \cong \operatorname{Im}(\phi) \leqslant S_{n}$, and so $N$ has index $m$ in $G$ where $m \leqslant n$ ! and $m$ divides $n$ !.

Lemma 4.3. If $H \leqslant_{f} G$ and $G$ is finitely generated, then $\exists K \leqslant_{f} H$ which is characteristic in $G$.

Proof. Suppose $H \leqslant{ }_{n} G$. We first show that there exist only finitely many subgroups of $G$ of a given index. As in the previous lemma, right multiplication by $G$ on $H \backslash G$ gives a homomorphism $\phi_{H}: G \rightarrow S_{n}$. Note that $\operatorname{Stab}(H)=H$ since $g \in \operatorname{Stab}(H) \Leftrightarrow H g=H$. Thus, by choosing $1 \in \mathbb{Z}_{n}$ to correspond to the coset $H$ in $H \backslash G$, the preimage of $\operatorname{Stab}(1)$ in $S_{n}$ is $H$. Hence $H=H^{\prime} \Leftrightarrow \phi_{H}=\phi_{H^{\prime}}$.

But $G$ finitely generated $\Rightarrow \exists$ only finitely many homomorphisms $G \rightarrow S_{n}$ (there are $(n !)^{|S|}$ maps from $S$ to $S_{n}$ ) and so there can only be finitely many index $n$ subgroups. Now let

$$
K:=\bigcap_{\phi \in \operatorname{Aut}(G)}(H) \phi
$$


and note that, for any $\phi \in \operatorname{Aut}(G),(H) \phi \leqslant_{n} G$. But there are only finitely many possible images for $H$ in (5), and so (since the intersection of finitely many subgroups of finite index is of finite index) $K$ is finite index in $G$. Finally, $K$ is characteristic in $G$ since the image of $K$ under $\psi \in \operatorname{Aut}(G)$ is contained within

$$
\bigcap_{\phi \in \operatorname{Aut}(G)}((H) \phi \psi)
$$

which is equal to $K$.

Lemma 4.4. MS14, Lem 2.2(ii)] Let $D$ be a group with the $R_{\infty}$ property and

$$
1 \longrightarrow D \longrightarrow E \longrightarrow F \longrightarrow 1
$$

be a short exact sequence of groups. If $D$ is characteristic in $E$ and $F$ is any finite group, then $E$ has the $R_{\infty}$ property.

Combining the previous two results provides an easier condition to check in order to show that all commensurable groups have the $R_{\infty}$ property.

Lemma 4.5. Let $G$ be a finitely generated group. If $G$ and all finite index subgroups of $G$ have the $R_{\infty}$ property, then all groups commensurable to $G$ have the $R_{\infty}$ property.

Proof. Let $H$ be commensurable to $G$. Then $\exists N \unlhd_{f} G, H$. By Lemma 4.3, there exists a group $U$ which is characteristic in $H$ and such that $U \leqslant_{f} G, H$. From our assumption that all finite index subgroups of $G$ have the $R_{\infty}$ property, $U$ has the $R_{\infty}$ property. Hence, by Lemma 4.4, $H$ has the $R_{\infty}$ property.

Our final aim is the following. Although it was done independently, our argument has similarities to [GP16, First proof of Thm. 3.8]. Their argument produces elements of different orders, whilst we produce elements of different cycle types. The flexibility that this affords allows our arguments to generalise from $H_{n}$ to certain subgroups $U_{p} \leqslant H_{n}$.

Theorem 4.6. Let $n \in \mathbb{N}$. If $G$ is any group commensurable to $H_{n}$, the $n^{\text {th }}$ Houghton group, then $G$ has the $R_{\infty}$ property.

Proof. We first work with FAlt. If $G$ is commensurable to $\operatorname{FAlt}(X)$, then there exists $N \unlhd_{f} \operatorname{FAlt}(X), G$. Now, since FAlt $(X)$ is simple and infinite, $N=\operatorname{FAlt}(X)$. Hence we have the short exact sequence

$$
1 \longrightarrow \operatorname{FAlt}(X) \longrightarrow G \longrightarrow F \longrightarrow 1
$$

where $F$ is some finite group. Let $\phi \in \operatorname{Aut}(G)$ and consider $\operatorname{FAlt}(X) \cap(\operatorname{FAlt}(X)) \phi$. This has finite index in FAlt $(X)$. Using Lemma 4.2 and that FAlt $(X)$ is simple, we have $(\operatorname{FAlt}(X)) \phi=\operatorname{FAlt}(X)$ i.e. that $\operatorname{FAlt}(X)$ is characteristic in $G$. Since FAlt $(X)$ is torsion, Corollary 3.7 states that it has the $R_{\infty}$ property. Hence Lemma 4.4 applies to $G$ implying that $G$ has the $R_{\infty}$ property.

We now work with $n \geqslant 2$. From Lemma 4.5, it is sufficient to show that, for any $n \geqslant 2$, all finite index subgroups of $H_{n}$ have the $R_{\infty}$ property.

Fix an $n \geqslant 2$. There are a family of finite index, characteristic subgroups of $H_{n}$ defined in BCMR16] and denoted $U_{p}$ where $p \in \mathbb{N}$. They showed that, for any $U \leqslant f H_{n}$, there exists a $p \in \mathbb{N}$ such that $U_{p} \leqslant{ }_{f} U$. This was strengthened in 
Cox17, Prop. 5.12] by showing that, for any $U \leqslant_{f} H_{n}$, there exists an $m \in \mathbb{N}$ such that $U_{m} \leqslant f U$ and

$$
\operatorname{Aut}(U)_{\Psi} \cong N_{\operatorname{Sym}\left(X_{n}\right)}(U) \leqslant N_{\operatorname{Sym}\left(X_{n}\right)}\left(U_{m}\right) \cong_{\Psi} \operatorname{Aut}\left(U_{m}\right)
$$

where $\Psi: N_{\operatorname{Sym}\left(X_{n}\right)}(G) \mapsto \operatorname{Aut}(G)$ is defined by $(g) \Psi=\phi_{g}$. Furthermore, by Cox17, Lem. 5.9], there is a monomorphism $\mu: N_{\operatorname{Sym}\left(X_{n}\right)}\left(U_{m}\right) \hookrightarrow N_{\operatorname{Sym}\left(X_{n m}\right)}\left(H_{n m}\right)$ and, for any $k \geqslant 2, N_{\operatorname{Sym}\left(X_{k}\right)}\left(H_{k}\right)=H_{k} \rtimes S_{k}$. Importantly, this monomorphism preserves cycle type. We shall apply Proposition 3.4 to show that any group with automorphism group contained within $N_{\mathrm{Sym}\left(X_{k}\right)}\left(H_{k}\right)$ for some $k \geqslant 2$ has the $R_{\infty}$ property.

Fix a $k \geqslant 2$. Notice that for all $r \in \mathbb{N} \backslash\{1\}$ and for all $g \in H_{k}, \eta_{r}(g)$ is finite. Given a $\rho \in H_{k} \rtimes S_{k}$, which is isomorphic to $\operatorname{Aut}\left(H_{k}\right)$ via the map $\rho \mapsto$ $\phi_{\rho}$, we have that $\eta_{r}(\rho)$ is infinite if and only if $\rho$ induces a cyclic permutation of $r$ branches of $X_{k}$. Thus, for all $\rho \in N_{\operatorname{Sym}\left(X_{k}\right)}\left(H_{k}\right)$ and all $r>k$ we have that $\eta_{r}(\rho)$ is finite. Now, for any $U \leqslant_{f} H_{n}$, there exists an $m \in \mathbb{N}$ such that $N_{\operatorname{Sym}\left(X_{n}\right)}(U) \leqslant N_{\operatorname{Sym}\left(X_{n}\right)}\left(U_{m}\right)$. Consider if $\rho \in N_{\operatorname{Sym}\left(X_{n}\right)}\left(U_{m}\right)$. Using the above homomorphism $\mu: N_{\operatorname{Sym}\left(X_{n}\right)}\left(U_{m}\right) \rightarrow N_{\operatorname{Sym}\left(X_{n m}\right)}\left(H_{n m}\right)$, we have that $\eta_{r}((\rho) \mu)$ is finite for all $r>n m$. Since $\mu$ preserves cycle type, $\eta_{r}(\rho)$ is also finite for all $r>n m$. Hence, by Proposition 3.4 $R\left(\phi_{\rho}\right)=\infty$ and so all automorphisms of $U$ have infinite Reidemeister number. Thus all finite index subgroups of $H_{n}$ have the $R_{\infty}$ property and so Lemma 4.5 yields the result.

\section{REFERENCES}

[BCMR16] J. Burillo, S. Cleary, A. Martino, and C. E. Röver, Commensurations and Metric Properties of Houghton's Groups, Pacific Journal of Mathematics, 285(2) (2016), 289301, MR 3575568

[BH16] T. Banakh and H. Mildenberger, Cardinal invariants distinguishing permutation groups, European Journal of Mathematics, 2 (2016), 493-507, MR 3498994

[Cam99] Peter J. Cameron, Permutation groups, London Mathematical Society Student Texts, vol. 45, Cambridge University Press, Cambridge, 1999, MR 1721031

[Cox17] C. G. Cox, Twisted Conjugacy in Houghton's groups, Journal of Algebra, 490 (2017), 390-436, MR 3690340

[DM96] John D. Dixon and Brian Mortimer, Permutation groups, Graduate Texts in Mathematics, vol. 163, Springer-Verlag, New York, 1996, MR 1409812

[Fel10] Alexander Fel'shtyn, New directions in Nielsen-Reidemeister theory, Topology Appl. 157 (2010), no. 10-11, 1724-1735, MR 2639839

[GP16] D. Gonçalves and S. Parameswaran, Sigma theory and twisted conjugacy-II: Houghton groups and pure symmetric automorphism groups, Pacific Journal of Mathematics, 280(2) (2016), 349-369, MR 3453976

[Hou78] C. H. Houghton, The first cohomology of a group with permutation module coefficients, Archiv der Mathematik 31 (1978), 254-258, MR 521478

[HNN49] Graham Higman, B. H. Neumann, and Hanna Neumann, Embedding theorems for groups, J. London Math. Soc. 24 (1949), 247-254. MR 0032641 (11,322d)

[HO16] M. Hull and D. Osin, Transitivity degrees of countable groups and acylindrical hyperbolicity, Israel Journal of Mathematics (Oct. 2016), 216(1), 307-353, MR 3556970

[JLS17] J. H. Jo, J. B. Lee, and R. L. Sang, The $R_{\infty}$ property for Houghton's groups, Journal "Algebra and Discrete Mathematics", Vol. 23 (2017), no. 2, 249-262, MR 3668980

[MS14] T. Mubeena and P. Sankaran, Twisted conjugacy classes in abelian extensions of certain linear groups, Canad. Math. Bull. 57 (2014), no. 1, 132-140 MR 3150726

[Ols82] A. Yu. Olshanskii, Groups of bounded period with subgroups of prime order, Algebra and Logic 21 (1983), 369-418; translation of Algebra i Logika 21 (1982), 553-618.

Mathematical Sciences, University of Southampton, SO17 1BJ, UK

E-mail address: cpgcox@gmail.com 